\title{
Plasmonics: A Path to Replace Electronics and Photonics by Scalable Ultra-fast Technology
}

\author{
Mallikarjun G Hudedmani*, Bindu Suresh Pagad \\ Department of Electrical and Electronics Engineering, KLE Institute of Technology, Opposite to Airport, \\ Gokul, Hubballi, Karnataka, India
}

${ }^{*}$ Corresponding Author email: mallikarjunh@yahoo.com

\section{Article History}

Received: 24 July 2019

Revised: 03 October 2019

Accepted: 21 October 2019

Published: 27 October 2019

\section{Student(s)}

\section{- $\quad$ Bindu Suresh Pagad}

Academic Year: 2018-19

Course Level: Bachelor

Course Name: BE

Course year: VIII ${ }^{\text {th }}$ Semester

$\operatorname{Mentor}(s)$

- Mallikarjun G Hudedmani

\begin{abstract}
Semiconductor devices, circuits, and components are dependent upon miniaturization for transporting huge amounts of data at a high speed these provide the ability to control the transport and storage of electrons. Current communication systems are based on either electrons or photonics. These modern electronic devices for information processing and sensing are functioning almost close to their fundamental speed and bandwidth limitations which a serious problem. The performance of electronic circuits, as well as photonics, is now becoming rather limited when digital information needs to be sent from one point to another. Plasmonics is a new technology a kind of photonics-based on surface plasmons viable. Surface plasmons are a way of guiding light. Surface Plasmon (SP) based circuits, which merge electronics and photonics at the nanoscale, may offer a solution to the size-compatibility problem. Optical fiber communication (OFC) is a wellknown light enabled information transmission mechanism communicates very effectively over large distance. Surface plasmons, on the other hand, can guide light only over distances of tens or hundreds of microns. Surface plasmons are the electromagnetic (optical) waves get generated from the interaction between light and the mobile conduction electrons on the surface of a metal. The surface plasmons created by the interaction of light near the surface possess unique advantages like the high speed of communication which is very essential for the current generation of electrical and medical fields.
\end{abstract}

Keywords: Plsmonics, Plasmons, Photonics, Ultrafast

\section{Introduction}

Today's state of the art microprocessors uses ultrafast transistors with dimensions of the order $50 \mathrm{~nm}$ but still they face challenge in carrying information to few centimetres long. From the literature from past 2 years, delay of interconnects faced by the transistor put substantial limitation. Optical interconnects such 
as fibre optic cables possess an ability to carry digital data at a very high speed like 1000 times that of electronic interconnects. Unfortunately, fibre optic cables are dimensionally 1000 times larger in comparison with electronic components. Different technologies have emerged to combine in the same circuit. However, the bulkiness imposes limitations. The plasmonic technology which is electromagnetic waves that propagate along the surface of a conductor finds its way in this application. The keyword Plasmonics is derived from 'plasmons' which refers to the quanta associated with longitudinal waves propagating in matter through the collective oscillation of electrons. Plasmon is the quasi particle resulting quantization of plasma oscillations just as photons and phonons are quantization of light and sound waves, respectively at optical frequencies. The comparison of need of energy to receive and send a surface plasmon pulse to electric charging of a metallic wire is very low. This could allow plasmons to travel along nano scale wires carrying information from one part of a microprocessor to another with a high bit rate [1]. Plasmonics forms a major part of the fascinating field of nano photonics, which explores how electromagnetic fields can be confined over dimensions on the order of or smaller than the wavelength. It is based on interaction between electromagnetic radiation and electrons under conduction at metals leading to an enhanced optical near field of sub-wavelength dimension. Research in this area demonstrates how a distinct and often unexpected behaviour can occur (even with for modern optical studies seemingly uninteresting materials such as metals) if discontinuities or sub wavelength structure is imposed. However, history has shown that despite the fact that the two main ingredients of plasmonics surface plasmon polaritons and localized surface plasmons have been clearly described as early as 1900, it is often far from trivial to appreciate the interlinked nature of many of the phenomena and applications of this field. This is compounded by the fact that throughout the $20^{\text {th }}$ century, surface plasmon polaritons have been rediscovered in a variety of different contexts. Plasmonics technology exploits the unique optical properties of manipulation of light at the nano scale. A greater synergy can be attained by integrating plasmonic, electronic, and conventional dielectric photonic devices on the same chip [2]. The plasmonic interconnects are the great boon for designers and developers to develop smaller and faster transistors. Surface plasmons can be excited on a flat nano film, nano-strip or other shaped nano particles such as nano sphere, nano-rod, nano cube and nano-star. The nano particles which are used to excite the surface plasmons by the application of light are known as localised surface plasmons. Silver and gold are of particular interest due to their high field enhancement and resonance wavelength lying in the visible spectral regime. The surface plasmons possess the speed equal to that of light as shown in Fig 1 and the wavelength tens of nano meters [3].

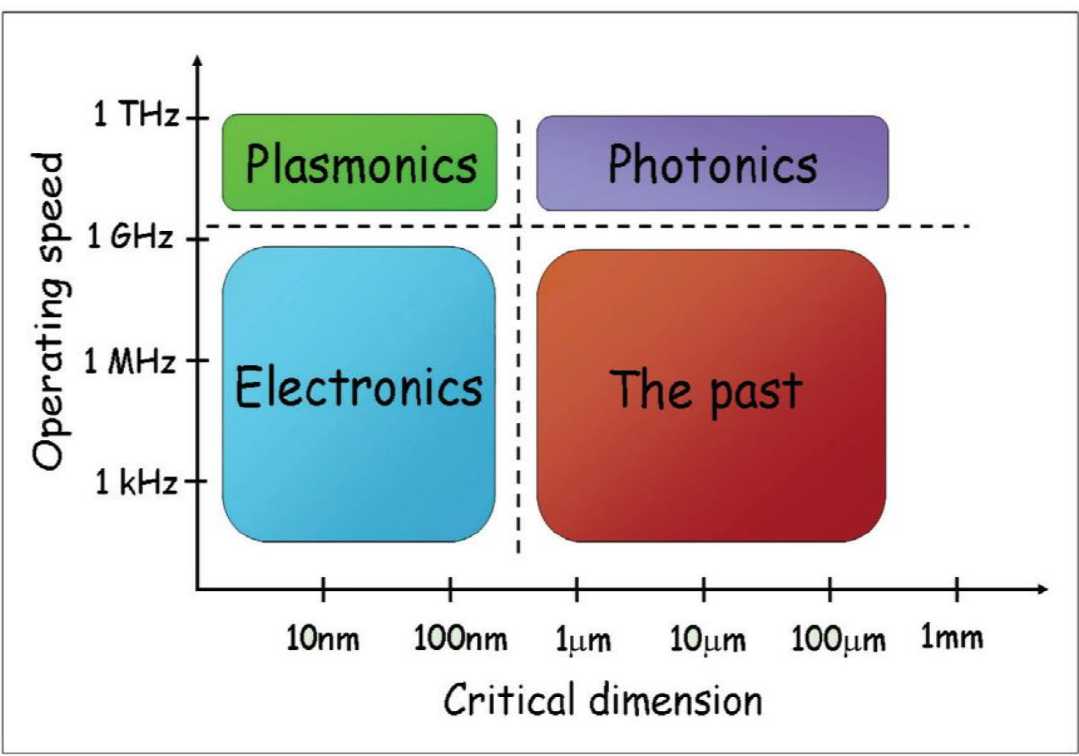

Figure 1: Operating speeds and sizes of plasmonics and other devices

The present growth and development in chip scale electronics and photonics has shown remarkable data processing and transport capabilities that permeate almost every facet of our lives. Plasmonics is an exciting new technology exploits the unique optical properties of metallic nanostructures to enable routing and manipulation of light at the nano scale [4]. Plasmonics is increasingly touted as a solution to replace traditional photonics. This is indeed a new generation of passive and active plasmonic elements are 
emerging. These plasmonic elements feature a tiny footprint with unlimited bandwidth in comparison to photonics. Plasmonic devices also suffer from high losses. The question then is where and when plasmonics really can make a difference [5]. The latest generation of plasmonic devices encompassing plasmonic electro optical modulators, detectors, RF-receivers, couplers and waveguides and compare them against devices relying on the traditional silicon technology.

\section{Plasmons and Plasmonics}

The plasmonics technology fits the electromagnetic waves into minuscule structures to yield a new generation of superfast computing chips and ultrasensitive molecular detectors. The plasmonics is growing very rapidly and has started to provide a whole range of exciting new research and development opportunities that go well beyond chip scale components. Presently we are witnessing an explosive growth in both the number and range of plasmonics applications. It is becoming eminently clear that both new fundamental science and device technologies are being enabled by the current plasmonics revolution. It was believed that light waves could not interact with any particle whose wavelength is smaller than their own. But the study of plasmons brings to light that this was a myth, since researchers have found that light's interaction with nano particles produce quasi particles called plasmons. Thus plasmons are collective oscillations of the free electron gas density at optical frequencies [6]. A plasma polariton is formed by the coupling of plasmons a photon which is quasi-particle. Since plasmons are the quantization of classical plasma oscillations, these are mathematically supported by Maxwell's equations. Since plasmons are density wave of electrons, created by interaction of light and metals, they are generated at optical frequencies and are small and rapid. They are said to embody the strongest points of both optical and electronic data transmission, allowing high speed data transfer. Plasmonic observed when the light beam striking a metal surface creates plasmons, electron density waves which can carry huge amounts of data. If the focus is on a surface etched with circular groove the beam produces concentric waves organizing electrons into high- \& low-density rings as presented in Fig 2.

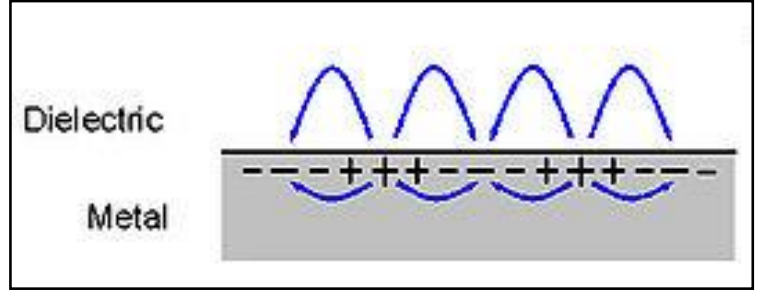

Figure 2: Movement of electrons

Presently, electronics plays an important role in communication and plasmonics started replacing electronics where a high data transfer rate is required. When the frequency of an electronic pulse increases, the electronic device suffers and becomes hot and wires become very loose. Hence by the principle, higher the frequency higher the data transfer rate but a huge amount of data cannot be transferred. On the other hand, when the size of an electronic wire reduces, its resistance increases but the capacitance remains almost the same. This leads to time delay effects. In photonics, optical fibres are used to transmit light along their axis by the process of total internal reflection but the size of the optical cable larger in terms of few hundreds of nanometres [7]. From the characteristics the propagation lengths for plasmonic waveguides are too short to propagate. The greater enhancements in propagation length can be obtained by careful engineering actions in which metal optical antenna should be used like scaled-down versions of a car antenna.

The field concentrating property of optical antennas may help to shorten the large gap between micro scale dielectric photonic devices and nano scale electronics. Fig3 shows details of a chip on those optical signals are routed through conventional dielectric optical wave guides. The mode size of such waveguides is larger than the underlying complementary metal oxide semiconductor (CMOS) electronics. Antenna helps to concentrate the electromagnetic signals and inject it into a nano scale photo detector. Due to the small size of the detector a small capacitance, low noise and

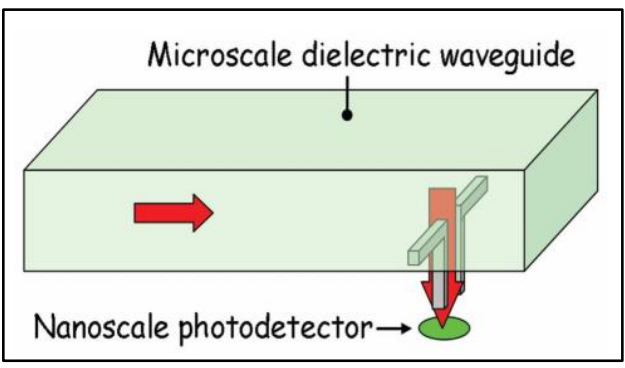
high speed of operation is observed [8-9].

Figure 3: Schematic of a nano scale antenna structure 


\subsection{Components of Plasmonics}

The main components of plasmonics are namely, Surface Plasmon Polaritons (SPP) and Localised Surface Plasmons (LSP). Surface plasmon polaritons (SPPs) are the special type of light waves or electromagnetic waves propagating along the metal dielectric interface and they are linked to the free electrons in the metal. The plasmonic waveguides is the metallic interconnect which support the waves thus serve as tiny optical waveguides. The motion of the light beam perpendicular to the metal interface has created a scope to interface nano scale electronics with similarly sized optical (plasmonic) devices. Presently silicon based integrated circuit technology already used nano scale metallic structures such as copper $(\mathrm{Cu})$ and aluminium $(\mathrm{Al})$ interconnects to route electronic signals between transistors on a chip. The plasmonic waveguides perform a dual function of carrying both optical and electrical signals and give rise to exciting new capabilities.

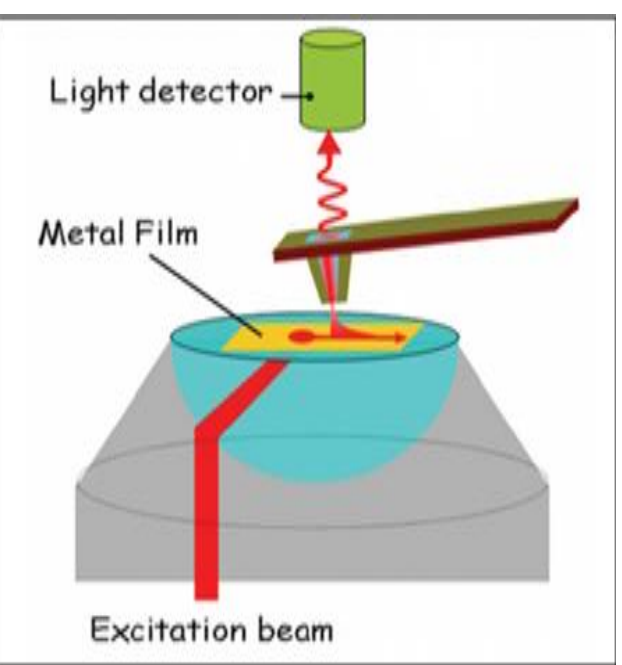

Figure 4: The red arrow shows how an SPP is launched from an excitation spot onto a metal film surface.

Localized surface plasmon (LSP) is the outcome of arrangement surface plasmon in a nano particle as shown in Fig 4. The LSP has two important properties namely, the electric field near the particle's surface is greatly enhanced and at the plasmon resonant frequency the particle's optical absorption is at its maximum [10]. The enhancement falls off quickly with distance from the surface and for noble metal nano particles, the resonance occurs at visible wavelengths and the near-infrared and mid-infrared region maximum optical absorption is noticed. Localized surface plasmon resonances (LSPRs) are the nonpropagating counterpart of SPPs, which can be excited in nanometer sized sub wavelength metallic particles. The electromagnetic field due to enhanced polarizability of the particle at certain frequency cause resonantly excited free electron cloud. The hot spots are the strongly enhanced near fields close to the metal surface created due to enhanced polarizability.

Plasmons are instrumental in applications to ultrasensitive optical detection, bio sensing, spectral photometry, light harvesting, photo catalysis, quantum optics, nonlinear photonics and meta materials. Current developments are focusing on reduction of electrons participating in the plasmons, thus unveiling new physics and generating wonderful applications like light modulation and sensing at the nano scale. Recently, electronics plays a very important role in communication. In spite of that, photonics has started replacing electronics where a high data transfer rate is required. As the frequency of an electronic pulse increases, the electronic device experience heating effect and wires become very loose. Hence according to the principle of the higher the frequency, the higher the data transfer rate, a huge amount of data cannot be transferred from one place to another. When the cross-sectional area of an electronic wire reduces its resistance increases but the capacitance remains almost the same. This leads to time delay effects. In photonics, optical fibres (cylindrical dielectric/non-conducting waveguides) are used. Total internal reflection helps in transmitting light along their axis. The fibre consists of a core surrounded by a cladding layer which is dielectric in nature. The refractive index of core must be large compared to cladding for the proper signal transfer [11]. The lateral confinement size of the optical cable is approximately half the wavelength of the light used so that the size of the optical cable is of the order of hundreds of nano meters larger than today's electronic devices.

Presently optical fibres are used worldwide for the communication systems use light signals that convey huge streams of voice communications and vast amounts of data. This gargantuan capacity has led some researchers to prophesy that photonic devices which channel and manipulate visible light and other electromagnetic waves could someday replace electronic circuits in microprocessors and other computer chips. Unfortunately, the diffraction limit imposes limitations size and performance of photonic devices. 
Plasmonic circuits present the potential to carry optical signals and electric currents through the same thin metal circuitry. Fig 5 and Fig 6 show demonstration of light on chip.

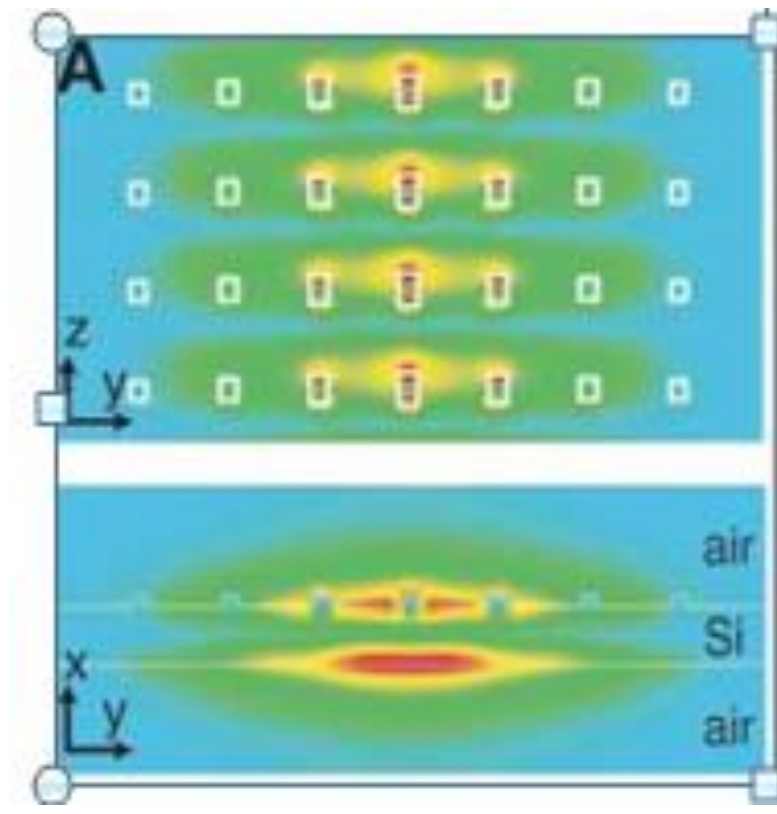

Figure 5: Simulation of electric field produced within the plasmon waveguide structure [12]

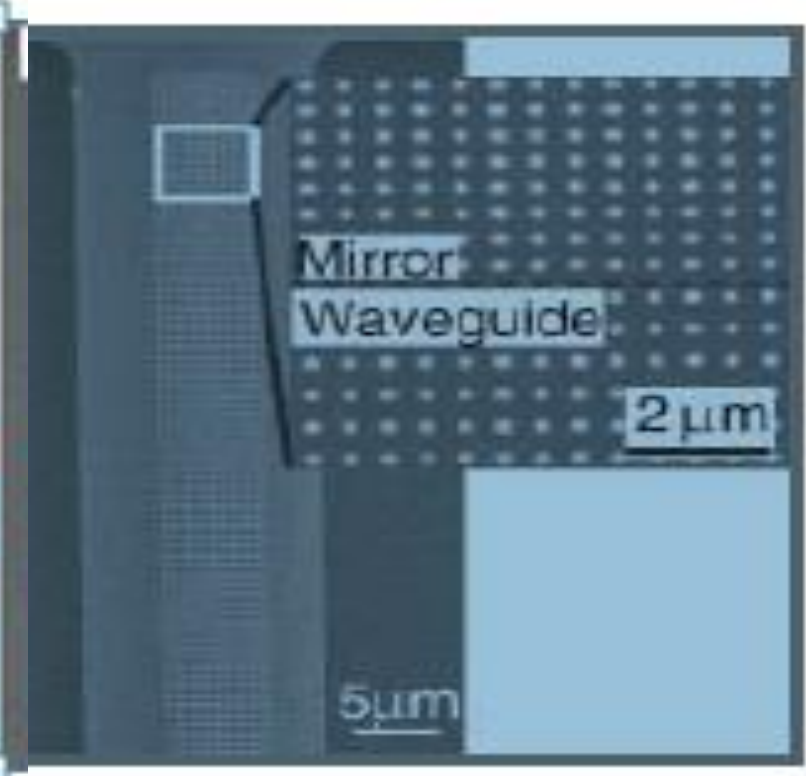

Figure 6: A plasmon waveguide consists of nano scale gold dots on a silicon-on-insulator surface [12]

The size limits the integration of optical and electronic circuits, but electronic circuits can be fabricated at dimensions below $100 \mathrm{~nm}$. On the other hand, the wavelength of light used in photonics circuits is on the order of $1000 \mathrm{~nm}$. Plasmonic chips will have plasmonic couplers and optical input and output ports for the interface. The couplers should have high conversion efficiency, along with a transmission length. The plasmonic interconnects may be used to solve the capacity problem in digital circuits when huge data needs to be transmitted from one section of a chip to another remote section. The data is converted to plasmonic information and sent along a plasmonic wire. Unfortunately, the current performance of plasmonic waveguides is insufficient for the said action. This initiates the urgent need for more work in this area [13].

\subsection{Communication with Plasmonics and Interconnect}

Electromagnetic waves at the nano scale experience greater control due to the plasmonic structures. Because of this, energy carried by plasmons allows for light localization in ultra-small volume far beyond the diffraction limit of light. To generate surface plasmons, it is necessary to excite the metal dielectric interface in which the dielectric constant of the metal is a function of frequency and negative. From the reviews of investigations, it is found that by the proper deign of metal dielectric interface, surface plasmons with the frequency as that of electromagnetic wave but of smaller wavelength is produced. This phenomenon could allow plasmons to travel along nano scale wires to carry information from one part to another. Owing to the ability of plasmonic waveguides to operate the spectrum ranging from visible to farinfrared region attract for the applications. A plasmon could travel several micrometres in the slot waveguide far enough to convey a signal from one part of a chip to another. The plasmon slot waveguide shrinks and squeezes the optical signal. Nano wires provide lateral confinement of the mode below the diffraction limit. These nano wires show larger attenuation than planar films but the movement of light by a distance of several microns. A set of variety of nano particles (namely, spheres and rods) can be used to transport EM waves from one nano particle to another using near field electro dynamic interaction [14]. The propagation distance depends upon the shape and nature of materials, separation between them as well as the dielectric constant of the host medium.

Optical interconnects must offer highest speed at lowest power consumption on an extremely compact footprint on a densely integrated chip. The fundamental challenges in realizing such optical interconnects 
are the transmitters that should be arranged in a highly parallel manner second, the fibre to chip interfaces that should be able to overcome distances from millimeters up to hundreds of meters. Plasmonic interconnects take leading edge shortly in the future integrated circuits. The physical limitations of conventional electric interconnects and the delay time for signal propagation is limited by an effective time constant typically dominated by the RC time of the interconnect itself. The energy required to transmit a bit through a length of interconnect is a good measure of the energy efficiency of communication on an integrated circuit. Consequently, the energy dissipated per unit length per bit transmission is weakly dependent on interconnect scale. As cross talk increases, bit error rates increase, so that interconnect themselves cease to be sufficiently reliable components in a fully operational integrated circuit.

At large operating frequencies typical of high-performance integrated circuits, the impedances between interconnect are reduced to values much lower than the resistance through which current must flow in the victim wire to counteract induced potential changes. As a result, the scaling trends of increasing frequency and increasing wire resistance lead to greater cross talk amongst wires. Local interconnects are too short to justify the addition of plasmonic sources and detectors. In addition to this, the reduction in length of local interconnects with standard scaling will mitigate most of the deleterious effects of reduced cross-sectional area. Global interconnects on the other hand, present plasmonic waveguides with strong competition from established technologies [15]. Conventional high index contrast dielectric waveguides offer the same bandwidth and latency advantages of plasmonic waveguides.

The long range plasmonic waveguides, which have propagation lengths suitable for global interconnects, tend to have transverse mode sizes akin to those of conventional dielectric waveguides. While it is possible that metallic waveguides may offer an advantage over dielectric waveguides if the materials systems are favorable to manufacturing, there is no substantial reduction in size associated with these modes. At the intermediate level however, the propagation lengths are short enough to employ plasmonic modes with cross sectional areas significantly below the diffraction limit. Of the standard metals used for plasmonic wave guiding (aluminum, gold and silver), silver is chosen to deal exclusively because it has the lowest loss across the frequency spectrum of interest [16]. An optimistic estimate of interconnect performance is given by ignoring surface scattering effects and attributing bulk material optical constants to the plasmonic waveguide.

\section{Features and Future of Plasmonics}

Few of the identified features of plasmonics are namely, they possess the frequency almost equal to that of light optical frequencies about 100000 times greater than the frequency of electronic microprocessors and the energy required to receive and send a surface plasmon pulse is lesser compared to that of electric charging of a metallic wire. This could allow plasmons to travel along nano scale wires carrying information with a high bit rate. These plasmons can be excited on a flat nano film, nano strip or other shaped nano particles such as nano sphere, nano rod, nano tube and nano star. The nano particles like Silver and Gold are of particular interest due to their high field enhancement and resonance wavelength falling in the visible spectral range. The speed of surface plasmons gets identifying in the soft x-ray range. The wavelength can be reduced by a factor of 10 relative to its free space value and still retaking the frequency. The ability to shrink the wavelength creates the path to nano scale plasmonic structures that could replace purely electronic circuits which use wires and transistors [17]. The plasmonics field is benefitted by the discovery of "meta materials" through which electron oscillations can result in exceptional optical properties. The latest development in producing plasmons to propagate through nano scale wires is to use complex waveguide geometries that can shrink the wavelength of the signal by squeezing it. A mass-production of minuscule plasmonic devices with a narrow dielectric stripe as a gap like lithography which is used to imprint circuit patterns on silicon chips may popularize the plasmonics. The frequency of an optical signal is much higher than that of an electrical one. More precisely 400,000 gigahertz versus 50 or 60 hertz. The resistance and capacitance effect of conventional electronics limit the data-carrying capacity of integrated circuits with electrical interconnects [18]. 
Hudedmani et al., Adv. J. Grad. Res.; Vol. 7, Issue 1, pp: 37-44, January 2020

The technology is rapidly evolving from discrete passive structures towards integrated active devices and revolutionize the bandwidth, speed, size, cost, power requirements of modern computational networks, enabling more efficient solutions to increasing complex problems. The future development in the next step is to integrate the components with an electronic chip and hence to demonstrate plasmonic data generation, transport and detection. Plasmon waves in the plasmonic technology on the metal behave similar to light waves in glass hence this concept helps in multiplexing. Plasmonic applications mainly depend on the cost of nanofabrication techniques and losses. The plasmonic nano circuits combine a high bandwidth with a high-level compaction and promising for all optical circuits. Plasmons can ferry data along computer chips. Plasmonic switches required for this are under development. Rotaxanes molecule is being used for this purpose [19].

\section{Conclusions}

Plasmonics has gained and developed an explosive growth past from few years. The knowledge base related to plasmonics is rapidly expanding. As a result, the plasmonic devices on a chip becoming clearly defined they were relatively slow and bulky in past. Plasmonics has the capability and future to play an important role in enhancing the processing speed of future integrated circuits. Besides creating new photonics devices, which are considerably smaller than the propagating light's wavelength, plasmonics is expected to be the key nanotechnology that will combine electronic and photonic components on the same chip. The advancements in semiconductor technology has brought an incredible change in scaling electronic devices to nano-scale dimensions. But the interconnect delay time issues are significant and still pose challenge towards the realization of purely electronic circuits operating above $10 \mathrm{GHz}$. In contrast, photonic devices possess a huge data carrying capacity. The dielectric photonic components are limited in their size by the laws of diffraction preventing scaling as in electronics. Finally, the review of plasmonics, its current state and future directions provide clarity that the Plasmonic devices find good coupling with similar speed photonic devices and similar size electronic components in the upcoming semiconductor developments as a revolution.

\section{Competing Interests}

The authors declared that no conflict of interest exist in this publication.

\section{How to Cite this Article:}

M. Hudedmani and B. Pagad, "Plasmonics: A Path to Replace Electronics and Photonics by Scalable Ultra-fast Technology", Adv. J. Grad. Res., vol. 7, no. 1, pp. 37-44, Oct. 2019. doi:10.21467/ajgr.7.1.37-44

\section{References}

[1] J. Leuthold, R. Bonjour, Y. Salamin, C. Hoessbacher, W. Heni, C. Haffner, A. Josten, B. Baeuerle, M. Ayata, A. Messner, U. Koch, T. Watanabe, Y. Fedoryshyn, P. Ma, M. Burla, D. L. Elder, and L. R. Dalton, "Plasmonics for Communications," in Optical Fiber Communication Conference, OSA Technical Digest (online) (Optical Society of America, 2018), paper M3G.2.

[2] Tong Zhang, Xuefeng Liu, Qin Chen, Jing Chen, and Haider Butt, "Plasmonics and Nanophotonics," Hindawi Publishing Corporation, Journal of Nanomaterials, vol. 2014, Article ID 746195, 1 page, 2014.

[3] Giovanni Manfredi, Preface to Special Topic: Plasmonics and solid state plasmasPhys. Plasmas 25, 031701 (2018).

[4] Mark L. Brongersma, Rashid Zia, and Jon Schuler, "Plasmonics- The Missing Link between Nanoelectronics and Microphotonics", Vol 3, Issue 3, page 360-362, 2007.

[5] Dmitri K. Gramotnev and Sergey I. Bozhevolnyi, "Plasmonics beyond the diffraction limit", Nature Photonics, Vol 4, page 83-91, Feb 2010.

[6] Mark I Stockman et al, “Roadmap on plasmonics”, Journal of Optics, J. Opt. 20 (2018) 043001, page 39, 2018.

[7] S. Nivedha, P. Ramesh Babu and K. Senthilnathan, "Surface plasmon resonance: physics and technology", Current Science a fortnightly journal of research, Vol 115, Issue 1, page 56-63, 10 $0^{\text {th }}$ July 2018.

[8] Viktoriia E. Babicheva, Nathaniel Kinsey, Gururaj V. Naik, Andrei V. Lavrinenko, Vladimir M. Shalaev, Alexandra Boltasseva, "Ultra-compact modulators based on novel CMOS-compatible plasmonic materials", Vol 21 Issue 22, page 27326-27337, 2013.

[9] Vandana Sharma, Shrishti Sharma, Roshan Jain, "Plasmonics Technology - A Review", World Journal of Research and Review (WJRR), Vol 1, Issue 2, Pages 06-08, December 2015

[10] Rishabh Chickballapur Nagaraj, Sativada Monica, Dr. M. N. Thippeswamy, “Plasmonics -A New Device Technology”, International Journal for Scientific Research \& Development, Vol 1, Issue 9, page 1870-1874, 2013. 
[11] E. P. Fitrakis, C. Kachris, A. Scandurra, A. Melikyan, M. Sommer, M. Kohl, J. Leuthold, V. Dolores-Calzadilla, M. K. Smit, D. Van Thourhout, Z. Hens, I. Suarez, J. Martinez-Pastor, I. Tomkos, 2012, "Plasmonic technology innovation: The chip-to-chip interconnect", NAVOLCHI, page 1-9, October 2012.

[12] Joel D. Cox and F. Javier Garci'a de Abajo, "Electrically tunable nonlinear plasmonics in graphene nanoislands", Nature Communications, page 1-8, December 2014.

[13] Ekmel Ozbay, "Plasmonics: Merging Photonics and Electronics at Nanoscale Dimensions" Science vol 13, pp189-193, 2006.

[14] Josep Miquel Jornet and Ian F. Akyildiz, "Graphene-based Plasmonic Nano-Antenna for Terahertz Band Communication in Nanonetworks", IEEE Journal on selected areas in communications/supplement-Part 2, Vol 31, Issue 12, page 685-694, December 2013.

[15] Nanfang Yu, Romain Blanchard, Jonathan Fan, Qi Jie Wang, Christian Pfl ugl, Laurent Diehl, Tadataka Edamura, Shinichi Furuta, Masamichi Yamanishi, Hirofumi Kan and Federico Capasso, “ Plasmonics for Laser Beam Shaping, IEEE Transactions on Nanotechnology", Vol 9, Issue 1, page 11-29, January 2010.

[16] Hassan M. G. Wassel, Daoxin Dai, Mohit Tiwari, Jonathan K. Valamehr, Luke Theogarajan, Jennifer Dionne, Frederic T. Chong, Timothy Sherwood, "Opportunities and Challenges of using Plasmonic Components in Nanophotonic Architectures", IEEE Journal on Emerging and Selected Topics in Circuits and Systems Vol 2, Issue 2, page 1-12, June 2012.

[17] F.Javier García de Abajo, "Graphene Plasmonics: Challenges and Opportunities", ACS photonics, Vol 1, page 135-152, 2014.

[18] Onur Tokel, Fatih Inci and Utkan Demirci, "Advance in Plasmonic Technologies for Point of Care Applications" Chem. Rev., Vol 114, page 5728-5752, 2014.

[19] Joseph R. Lakowicz, Joanna Malicka, Evgenia Matveeva, Ignacy Gryczynski, and Zygmunt Gryczynski, "Plasmonic Technology: Novel Approach to Ultrasensitive Immunoassays", July 2005.

Publish your books with AIJR publisher-

$\checkmark \quad$ Publish with ISBN and DOI.

$\checkmark$ Publish Thesis/Dissertation as Monograph.

$\checkmark$ Publish Book Monograph.

$\checkmark$ Publish Edited Volume/ Book.

$\checkmark$ Publish Conference Proceedings

$\checkmark \quad$ Retain full copyright of your books.

Submit your manuscript at books.aijr.org
Publish your research article in AIJR journals-

$\checkmark \quad$ Online Submission and Tracking

$\checkmark$ Peer-Reviewed

$\checkmark$ Rapid decision

$\checkmark \quad$ Immediate Publication after acceptance

$\checkmark$ Articles freely available online

$\checkmark \quad$ Retain full copyright of your article.

Submit your article at journals.aijr.in 